\title{
An integrated approach for reconstructing a surface model of the proximal femur from sparse input data and a multi-resolution point distribution model: an in vitro study
}

\author{
Guoyan Zheng • Steffen Schumann • \\ Miguel A. González Ballester
}

Received: 27 August 2008 / Accepted: 21 June 2009 / Published online: 24 July 2009

(C) CARS 2009

\begin{abstract}
Background Accurate reconstruction of a patient-specific surface model of the proximal femur from preoperatively or intraoperatively available sparse data plays an important role in planning and supporting various computer-assisted surgical procedures.

Methods In this paper, we present an integrated approach using a multi-resolution point distribution model (MR-PDM) to reconstruct a patient-specific surface model of the proximal femur from sparse input data, which may consist of sparse point data or a limited number of calibrated X-ray images. Depending on the modality of the input data, our approach chooses different PDMs. When 3D sparse points are used, which may be obtained intraoperatively via a pointer-based digitization or from a calibrated ultrasound, a fine level point distribution model (FL-PDM) is used in the reconstruction process. In contrast, when calibrated X-ray images are used, which may be obtained preoperatively or intraoperatively, a coarse level point distribution model (CL-PDM) will be used.

Results The present approach was verified on 31 femurs. Three different types of input data, i.e., sparse points, calibrated fluoroscopic images, and calibrated X-ray radiographs, were used in our experiments to reconstruct a surface model of the associated bone. Our experimental results demonstrate promising accuracy of the present approach.

Conclusions A multi-resolution point distribution model facilitate the reconstruction of a patient-specific surface model of the proximal femur from sparse input data.
\end{abstract}

G. Zheng $(\varangle) \cdot$ S. Schumann · M. A. González Ballester ARTORG Center for Biomedical Engineering Research, University of Bern, Stauffacherstrasse 78, 3014 Bern, Switzerland e-mail: guoyan.zheng@ieee.org
Keywords Reconstruction - Multi-resolution point distribution model $\cdot$ Statistical shape analysis

\section{Introduction}

A patient-specific surface model of the proximal femur plays an important role in planning and supporting various computer-assisted surgical procedures including total hip replacement, hip resurfacing, and osteotomy of the proximal femur. The common approach to derive 3D models of the proximal femur is to use imaging technique such as computed tomography (CT) or magnetic resonance imaging (MRI). However, the high logistic effort, the extra radiation associated with the CT-imaging, and the large quantity of data to be acquired and processed make them less functional. The alternative presented here is to reconstruct the surface model using sparse input data consisting of dozens of threedimensional (3D) surface points (e.g., 50 points) or a limited number of two-dimensional (2D) calibrated X-ray images (e.g., 2 to 4 images).

Constructing an accurate 3D surface model from sparse input data is a challenging task. Additionally, inherent to the navigation application is the high accuracy and robustness requirements. When surface reconstruction is used for the purpose of surgical guidance, it requires that the algorithm satisfies the following criteria: (a) accurate geometrical information about the underlying anatomical structure can be derived from the reconstructed surface model, (b) target reconstruction error of the reconstructed surface model should be in the range of surgical usability, which is typically in the area of $1.5 \mathrm{~mm}$ average error (2-3 mm worst case) [1], (c) $95 \%$ success rate is normally required, when an appropriate initialization is given [1], (d) minimal user interaction during data acquisition and algorithm execution 
is highly appreciated for a sterilized environment. One way to achieve these criteria is to build a point distribution model (PDM) and to adapt the model to the patient's individual anatomy using the input sparse input data-a procedure that is called statistical shape model instantiation and registration [2], or simply model instantiation.

The procedure of statistical instantiation can be divided into two sub-procedures: correspondence establishment and model fitting. In the former sub-procedure, the correspondences between the input data and the point distribution model are established. The point distribution model is then adapted to the patient's individual anatomy in the latter procedure based on the established correspondences and the input data.

The correspondence problem in point distribution model construction is getting more and more attentions [3-6]. Various groups [7-9] have conducted benchmark studies to compare different correspondence establishing methods for point distribution model construction. However, fewer attentions are paid to the correspondence problem in statistical instantiation. In the former case, where a dense correspondence has to be established between different training instances to generate a statistical shape model, constraints such as oneto-one mapping can be applied [6]. In the latter case, however, the correspondence assignment will not be a direct one-to-one mapping due to the sparsity of the input data. Furthermore, because of the fixed surface parameterization for the point distribution model, we argue that the correspondence assignment as used in the landmark sliding [10], where one component in the input data is assigned to an interpolated component in the point distribution model, or the soft assignment in robust point matching [11] should be avoided. We hypothesize that in statistical instantiation different formats of input data may need different correspondence establishing methods based on different point distribution models with different surface parameterization. To this end, we propose to solve the statistical instantiation problem for both 2D and 3D input data with an integrated approach combining sophisticated surface reconstruction techniques with a multi-resolution point distribution model (MR-PDM).

The paper is organized as follows. The second section reviews the related work. Third section presents the construction of the MR-PDM of the proximal femur. Fourth section describes the integrated approach combining our previous works on 3D-3D surface reconstruction [12-14] and those on 2D-3D surface reconstruction [15-17]. Experiments and results are presented in fifth section, followed by conclusions in the last section.

\section{Related works}

Statistical shape analysis [18-20] is an important tool for understanding anatomical structures from medical images.
A statistical model gives an effective parameterization of the shape variations found in a collection of sample models of a given population. Model based approaches [21-23] are popular due to their ability to robustly represent objects. Intraoperative reconstruction of a patient-specific model from sparse input data can be potentially achieved through the use of a statistical model. Statistical model building consists of establishing legal variations of shape from a training population. A patient-specific model is then instantiated through fitting the statistical model to intraoperatively acquired data. Thus, the aim of the statistical instantiation is to extrapolate from sparse input data a complete and accurate anatomical representation. This is particularly interesting for minimally invasive surgery (MIS), largely due to the operating theater setup.

Several research groups have explored the methods for reconstruction a patient-specific model from a statistical model and sparse input data such as digitized points [12-14, 24-27], a limited number of calibrated X-ray images [15-17, 28-32], or tracked ultrasound [2,33-36]. Except the method presented by Yao and Taylor [29], which depends on a deformable 2D/3D registration between an appearance based statistical model [37] and a limited number of X-ray images, all other methods have their reliance on a point distribution model (PDM) in common. In Fleute and Lavallée [24], a statistical shape model of the distal femur was fitted to sparse input points by simultaneously optimizing both shape and pose parameters. Their technology has been incorporated into a system for computer-assisted anterior cruciate ligament surgery and preliminary results were published in [25]. Chan et al. [2,33,34] used a similar algorithm, but optimized the shape and pose parameters separately. Tracked ultrasound was used as the input in their work to instantiate 3D surface models of the complete femur and pelvis from their associated statistical shape models. Following the seminal work of Blanz and Vetter for the synthesis of 3D faces using a morphable model [38], Rajamani et al. [26,27] incorporated a Mahalanobis prior for a robust and stable surface model instantiation. In our recent work [12-14], we proposed to use the dense surface point distribution model (DS-PDM) and a reconstruction scheme combining statistical instantiation and regularized shape deformation for an accurate and robust reconstruction of a patient-specific surface model of the proximal femur from dozens of points. This reconstruction scheme has also been combined with a novel 2D-3D correspondence establishing algorithm [15] for reconstructing surface model of the proximal femur from a limited number of calibrated X-ray images $[16,17]$.

\section{Construction of a multi-resolution point distribution model}

The MR-PDM used in this paper was constructed from a training database consisting of 30 proximal femoral surfaces 
Fig. 1 An example shows how to use Loop's scheme to subdivide a surface patch from a coarse resolution to a fine resolution. Left a surface patch to be subdivided. Right superimposition of the original surface patch (described by dashed, black lines and gray disks) on the subdivided surface patch (described by solid, red lines and red disks). Each triangle of the original surface path has been divided into four smaller triangles
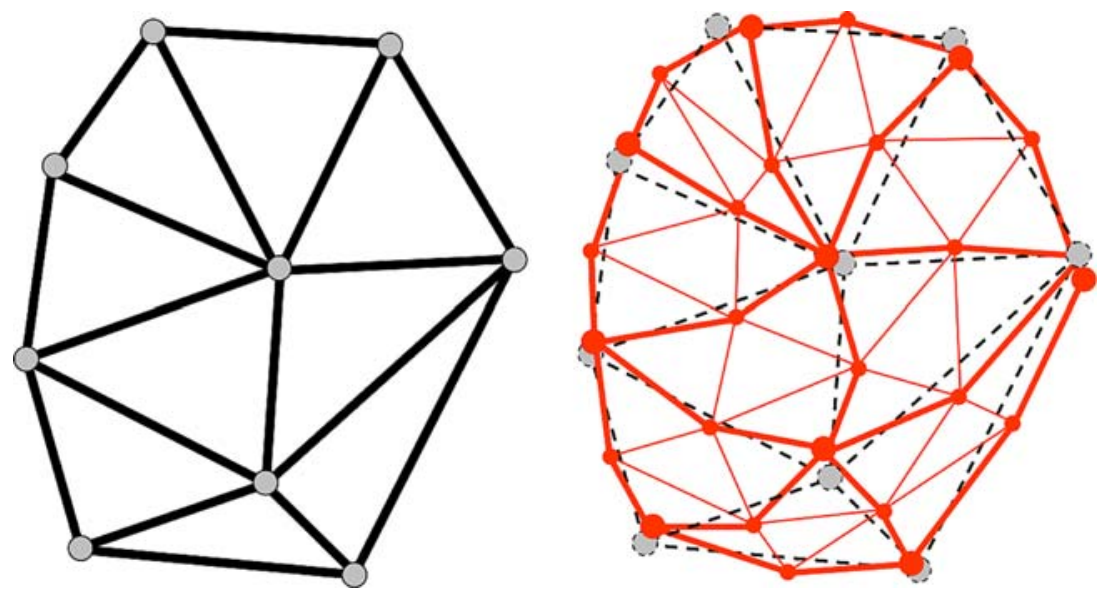

from above the lesser trochanter. In the coarsest level, a sequence of correspondence establishing methods presented in [7] was employed to optimally align surface models segmented from CT volume. It started with a manually initialized subdivision surfaces similar to Wang et al. [39] and then was optimized using minimum description length (MDL) based principle as proposed in $[3,40]$.

Following the alignment, the PDM in this level is constructed as follows. Let $\mathbf{x}_{i}=\left(p_{0}, p_{1}, \ldots, p_{N-1}\right)$, $i=0,1, \ldots, m-1$, be $m$ (here $m=30$ ) members of the aligned training surfaces. Each member is described by a vectors $\mathbf{x}_{i}$ with $N(N=4098)$ vertices:

$\mathbf{x}_{i}=\left\{x_{0}, y_{0}, z_{0}, x_{1}, y_{1}, z_{1}, \ldots, x_{N-1}, y_{N-1}, z_{N-1}\right\}$

The PDM is obtained by applying principal component analysis on these surfaces.

$\mathbf{D}=\frac{1}{(m-1)} \cdot \sum_{i=0}^{m-1}\left(\mathbf{x}_{i}-\overline{\mathbf{x}}\right) \cdot\left(\mathbf{x}_{i}-\overline{\mathbf{x}}\right)^{T}$

$\mathbf{P}=\left(\mathbf{p}_{0}, \mathbf{p}_{1}, \ldots, \mathbf{p}_{m^{\prime}-1}\right) ; \quad D \cdot \mathbf{p}_{i}=\sigma_{i}^{2} \cdot \mathbf{p}_{i}$

$\sigma_{0} \geq \sigma_{1} \geq \cdots \geq \sigma_{m^{\prime}-1}>0 ; \quad m^{\prime} \leq m-1$

where matrices $\overline{\mathbf{x}}$ and $\mathbf{D}$ represents the mean vector and the covariance matrix, respectively; $\left\{\sigma_{i}^{2}\right\}$ are non-zero eigenvalues of the covariance matrix $\mathbf{D}$, and $\left\{\mathbf{p}_{i}\right\}$ are the corresponding eigenvectors. The sorted eigenvalues $\sigma_{i}^{2}$ and the corresponding eigenvector $\mathbf{p}_{i}$ of the covariance matrix are the principal directions spanning a shape space with $\overline{\mathbf{x}}$ representing its origin. $m^{\prime}$ is a cut-off number for selecting the first $m^{\prime}$ th major eigenmodes.

Then, any one of the instance in this space can be expressed as:

$\mathbf{x}=\overline{\mathbf{x}}+\sum_{i=0}^{m^{\prime}-1} \alpha_{i} \mathbf{p}_{i}$
And the estimated normal distribution of the coefficients $\left\{\alpha_{i}\right\}$ is:

$p\left(\alpha_{0}, \alpha_{1}, \ldots, \alpha_{m^{\prime}-1}\right)=(2 \pi)^{-\frac{m^{\prime}}{2}} \cdot e^{-\frac{1}{2} \sum_{i=0}^{m^{\prime}-1}\left(\alpha_{i}^{2} / \sigma_{i}^{2}\right)}$

where $\sum_{i=0}^{m^{\prime}-1}\left(\alpha_{i}^{2} / \sigma_{i}^{2}\right)$ is the Mahalanobis distance defined on the distribution.

The vertices for constructing the denser point distribution model in a finer resolution are then obtained by iteratively subdividing the aligned surface model in the coarser resolution. The basic idea of subdivision is to provide a smooth limit surface model which approximates the input data. Starting from a mesh in a low resolution, the limit surface model is approached by recursively tessellating the mesh. The positions of vertices created by tessellation are computed using a weighted stencil of local vertices. The complexity of the subdivision surface model can be increased until it satisfies the user's requirement.

In this work, we use a simple subdivision scheme called Loop's scheme, invented by Loop [41], which is based on a spline basis function defined on a 3D mesh, called the threedimensional quartic box spline. The subdivision principle of this scheme is very simple. Three new vertices are inserted to divide a triangle in a coarse resolution to four smaller triangles in a fine resolution. Figure 1 shows how a surface patch is divided from a coarse resolution to a fine resolution using Loop's scheme.

Although the subdivision strategy is simple in Loop's scheme, the exact rules for computing the vertex positions in the fine resolution from the vertices in the coarse resolution depends on the local structure of a mesh, i.e., the valence of a vertex, which means the number of edges connected to a vertex. Depending on the valence of a vertex, Loop [41] proposed to use three different rules, as shown in Fig. 2. The goal is to guarantee that the limit surface of the scheme is smooth. The first rule, shown in Fig. 2, right, is used to compute the new positions of a regular vertex with a valence 6 from 
Fig. 2 Computing rules of Loop's scheme for regular vertices with a valence 6 , edge vertices, and extraordinary vertices with a valence $n$, where $a=\frac{5}{8}-\left(\frac{3}{8}+\frac{1}{4} \cos \left(\frac{2 \pi}{n}\right)\right)^{2}$
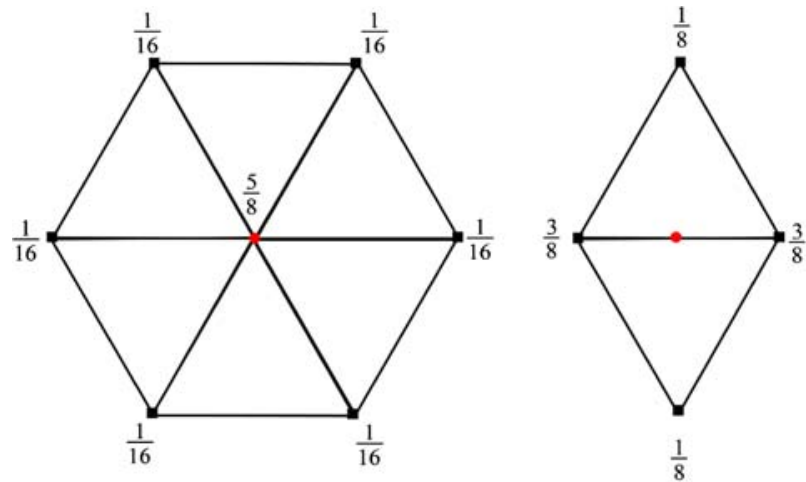

the previous resolution. The second rule, shown in Fig. 2, middle, is used to compute the points inserted at an edge. Please keep it in mind that this rule can be applied anywhere. And as shown in Fig. 1, all newly inserted vertices are regular vertices with a valence 6. The third rule, shown in Fig. 2, right, is used to compute the new position of an extraordinary vertex with a valence $n(n \neq 6)$ inherited from the previous level.

According to the computing rules of Loop's scheme, the positions of all vertices in a fine resolution are computed from a weighted stencil of local vertices in previous resolution. As the input surface models have been optimized for establishing correspondences, it is reasonable to conclude that the output models are also aligned. Principal component analysis can be applied on these dense surface models to establish a dense surface point distribution model (DS-PDM). In our previous work [12-14], we found that a single level subdivision is enough for our purpose. We thus created a two-level point distribution model (TL-PDM).

\section{An integrated surface model reconstruction approach}

Based on the two-level point distribution model, we developed an integrated surface model reconstruction approach which can seamlessly handle both 3D sparse points and a limited number of X-ray images. When a set of 3D points are used, the fine level point distribution model (FL-PDM) will be chosen, which facilitates the point-to-surface correspondence establishment. But if the input is a limited number of calibrated X-ray images, we will use the coarse level point distribution model (CL-PDM) to speed up the computation. For completeness, we will briefly present these two methods below. Details can be found in our previously published works [12-17].

\section{D-3D reconstruction method}

[12-14]: The 3D-3D reconstruction problem is formulated as a three-stage optimal estimation process. The first stage, affine registration, is to iteratively estimate the scale and the 6 degree-of-freedom rigid transformation between the mean shape of the PDM and the sparse input data using a correspondence building algorithm and a variant of iterative closest point (ICP) algorithm [42]. The estimation results of the first stage are used to establish point correspondences for the second stage, statistical instantiation, which optimally and robustly instantiates a surface model from the FL-PDM using a statistical approach [27]. The instantiated surface model is taken as the input for the third stage, regularized shape deformation, where the input surface is further deformed by an approximating thin-plate spline (TPS) based vector transform [43] to refine the statistically instantiated surface model.

\section{D-3D reconstruction method}

[15-17]: Our 2D-3D reconstruction approach combines statistical instantiation and regularized shape deformation as described above with an iterative image-to-model correspondence establishing algorithm [15]. The image-to-model correspondence is established using a non-rigid 2D point matching process, which iteratively uses a symmetric injective nearest-neighbor mapping operator and 2D thin-plate splines based deformation to find a fraction of best matched 2D point pairs between features detected from the calibrated $\mathrm{X}$-ray images and the projections of the apparent contours extracted from the $3 \mathrm{D}$ model. The obtained $2 \mathrm{D}$ point pairs are then used to set up a set of 3D point pairs such that we turn a $2 \mathrm{D}-3 \mathrm{D}$ reconstruction problem to a $3 \mathrm{D}-3 \mathrm{D}$ one, which can be solved by the 3D-3D reconstruction approach as described above, but using a CL-PDM.

\section{Experimental results}

We conducted experiments on 31 femurs ( 1 plastic femur and 30 cadaver femurs; note: none of them has been included for constructing the TL-PDM) with different shapes to validate the present approach. Three different types of input data, i.e., sparse points, calibrated fluoroscopic images, and cali- 
Fig. 3 Errors of reconstructing 3D surface models of seven cadaver femurs using clinically relevant 3D sparse point data

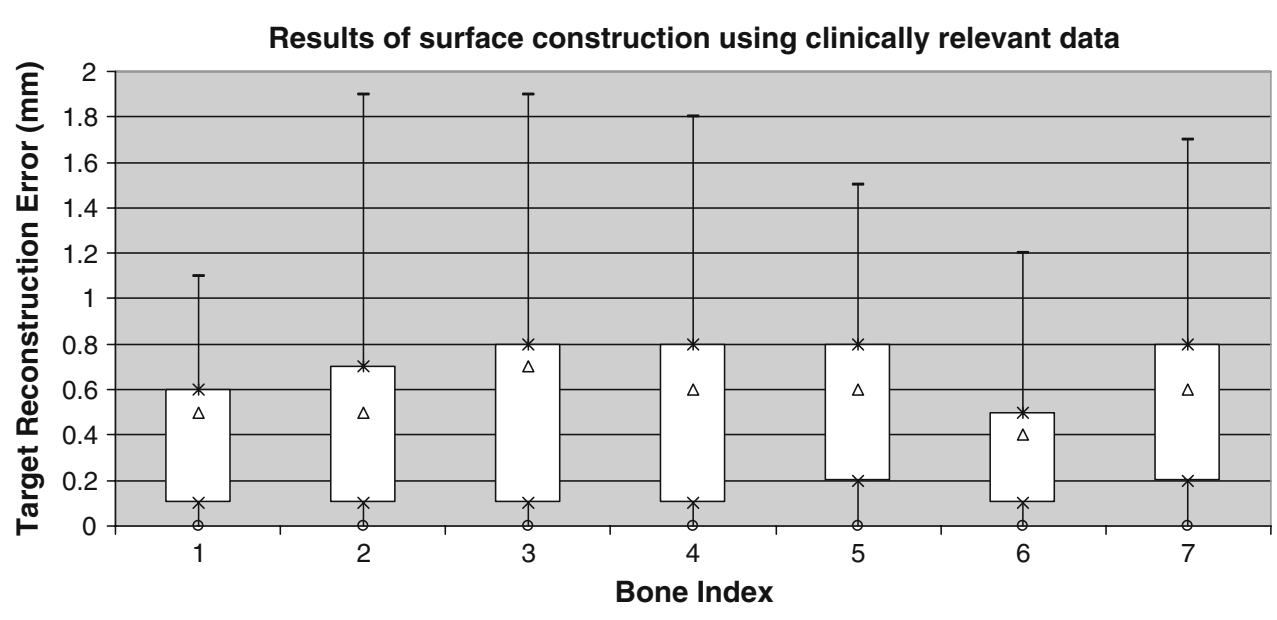

$\times$ First Quartile $\circ 5$ Percentile $\Delta$ Median - 95 Percentile $*$ Third Quartile brated X-ray radiographs, were used in our experiments to reconstruct the surface model of the associated bone.

\section{Reconstruction error measurement}

To quantify the reconstruction error in the cadaver femur experiment, a target reconstruction error (TRE) was used. The TRE is defined as the distance between the actual and the reconstructed position of selected target features, which can be landmark points or surface models.

Validation experiments and results

We designed and conducted following three experiments to validate the present approach.

(1) Reconstruction of surface models of seven cadaver femurs using clinically relevant sparse points:

Total hip replacement and hip resurfacing procedures operated with posterior approach were identified as the potential clinical applications. At one stage of such surgeries, after internal rotation and posterior dislocation of the hip, most of the femoral head, neck, and some part of trochanteric and intertrochanteric (crest and line) regions are exposed [44]. Obtaining sparse surface points from these intraoperatively accessible regions and reconstructing a patient-specific 3D surface model of the proximal femur with reasonable accuracy will be useful for the above mentioned surgeries. In this experiment, one set of 50 points was used to reconstruct the surface model of each cadaver bone and the other set consisted of 200 points was used to evaluate the reconstruction errors. The results of surface reconstruction using clinically relevant sparse points are presented in Fig. 3. For each case, the overall execution time was less than one minute
(2) Reconstruction of surface models of 11 cadaver femurs using a limited number of calibrated C-arm images:

In this experiment, two studies using different number of images were performed for each bone. In the first study two images acquired from anterior-posterior (AP) and lateralmedial (LM) directions were used to reconstruct the surface model of each cadaver femur. In the second one, an image acquired from oblique direction was additionally used together with the above mentioned AP and LM images. The reconstruction accuracies were evaluated by randomly digitizing 100-200 points from each surface of the cadaver specimen and then computing the distance from those digitized points to the associated surface reconstructed from the images. The median and mean reconstruction errors of both experiments are presented in Table 1. An average mean reconstruction error of $1.2 \mathrm{~mm}$ was found when only AP and LM images were used for each bone. It decreased to $1.0 \mathrm{~mm}$ when three images were used.

(3) Reconstruction of surface models of 23 femurs (1 plastic femur and 22 cadaver femurs) using a pair of calibrated $\mathrm{X}$-ray radiogaphs:

One plastic femur and twenty-two cadaver femurs were used in this experiment. Anterior and posterior views of part of the cadaver femurs are shown in Fig. 4.

For each bone, two calibrated X-ray radiographs were acquired. All X-ray radiographs were calibrated using a custom-made calibration cage $[45,46]$. Furthermore, two dry cadaver femurs were used together with a dry pelvis to construct an assembled hip. For each side of the assembled hip, two calibrated X-ray radiographs were acquired. Thus, totally we obtained 25 pairs of calibrated images. Each time, a pair of the calibrated images was used as the input to reconstruct the surface model of the associated bone. 
Table 1 Reconstruction errors when different number of images were used

\begin{tabular}{lllllllllllll}
\hline \multicolumn{1}{l}{ Reconstruction errors when only AP and LMere used for each bone } \\
Bone index & 1 & 2 & 3 & 4 & 5 & 6 & 7 & 8 & 9 & 10 & 11 \\
Median (mm) & 1.3 & 0.8 & 1.5 & 1.0 & 1.3 & 1.0 & 1.1 & 1.0 & 0.8 & 1.1 & 1.2 \\
Mean (mm) & 1.5 & 0.8 & 1.4 & 1.3 & 1.4 & 1.2 & 1.2 & 1.2 & 1.0 & 1.1 & 1.6 \\
Reconstruction errors when all three images & were used for each bone & & & & \\
Bone index & 1 & 2 & 3 & 4 & 5 & 6 & 7 & 8 & 9 & 10 & 11 \\
Median (mm) & 1.3 & 0.7 & 0.7 & 1.1 & 1.0 & 1.1 & 0.8 & 0.9 & 0.7 & 1.0 & 0.9 \\
Mean (mm) & 1.3 & 0.7 & 0.8 & 1.2 & 1.1 & 1.1 & 1.1 & 0.9 & 0.9 & 1.1 & 1.2 \\
\hline
\end{tabular}

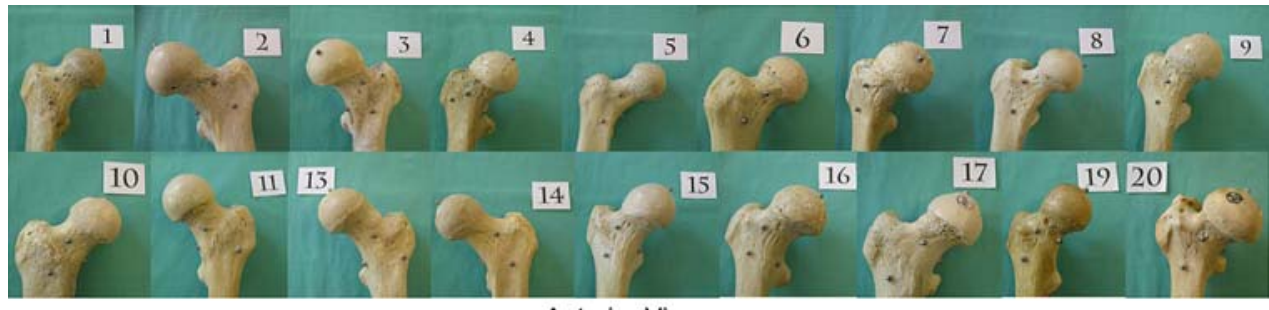

Anterior View

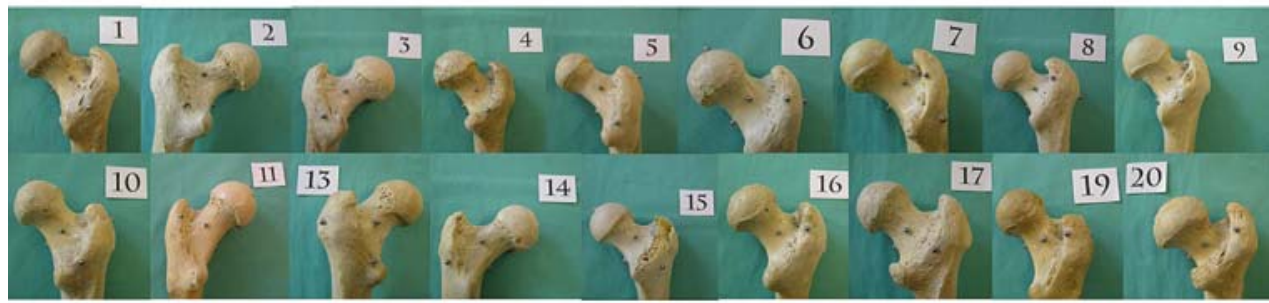

Posterior View
Fig. 4 The anterior view (top two rows) and the posterior view of part of the femurs used in the third experiment
To evaluate the reconstruction accuracy, we established the ground truths using two different methods. As 19 of them (including the plastic bone) are naked bones, we decided to use a hand-held laser-scan reconstruction method (T-SCAN, Steinbichler, Neubeuern, Germany) to obtain the surface model of these bones. The advantages of using such a method to obtain the ground truths include its high reconstruction accuracy (in the range of $0.1 \mathrm{~mm}$ according to the manufacturer) and the void of the segmentation errors which are inherent to the CT-scan reconstruction method. However, for the rest of four femurs, we had to use CT-scan reconstruction method to derive the ground truths as two of them are part of a wet cadaver pelvis and the other two are dry femurs but are glued with the associated acetabula. For this purpose, we used a commercially available software package, Amira 5.0 (TGS Europe, Paris, France), for semiautomatic segmentation of the surface models of those femurs. The derived ground truths were transformed to the associated reference coordinate systems of the reconstructed surface models by performing a surface-based rigid registration [42]. After that, the open source tool MESH [47] was used to compute the distance between the surface model reconstructed from each pair of X-ray images and its associated ground truth. The errors of reconstructing surface models using all 25 pairs of images are shown in Fig. 5. It was found that the mean errors of reconstructed surface models in comparison to their associated ground truths ranged from 0.6 to $1.4 \mathrm{~mm}$ and the root mean square (RMS) errors ranged from 0.8 to $1.9 \mathrm{~mm}$. The average mean error was found to be $0.95 \mathrm{~mm}$. Such accuracy was regarded as accurate enough for surgical navigation applications according to Livyatan et al. [1]. A reconstruction example and its associated error distribution are shown in Figs. 6 and 7, respectively.

\section{Discussions and conclusions}

We have presented an integrated approach using the MRPDM for robust and accurate anatomical shape reconstruction from sparse input data. Based on the modalities of the input data, the point distribution model of an appropriate resolution was used. In this approach, the 3D-3D reconstruction problem is formulated as a three-stage optimal estimation process. In each stage, the best result is optimally estimated under the assumption for that stage. The FL-PDM is employed in all stages to facilitate the correspondence establishment. When a limited number of calibrated X-ray images are used, the CL-PDM is employed to speed up the computation. A 2D-3D correspondence establishing algorithm based on a non-rigid $2 \mathrm{D}$ point matching process is 
Fig. 5 Error of reconstructing surface models from all 25 pairs of images

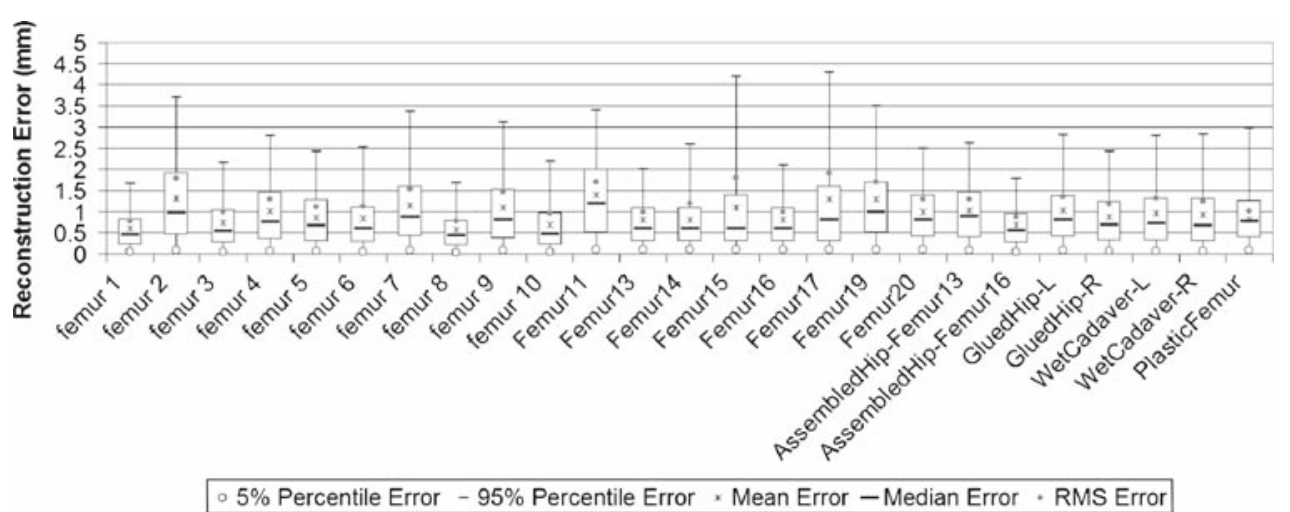

B
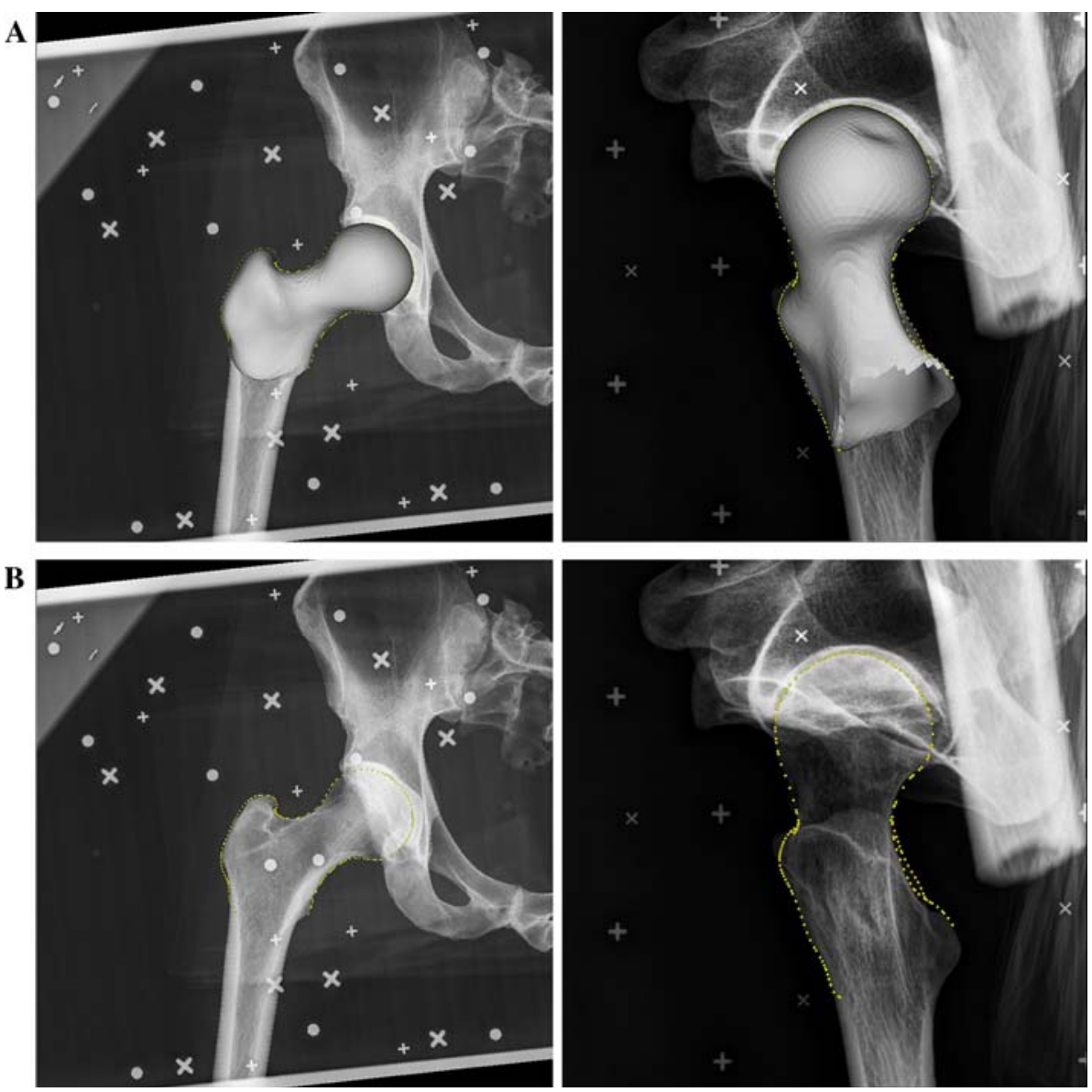

Fig. 6 A surface model of a cadaver femur reconstructed from calibrated X-ray radiographs by our $2 \mathrm{D}-3 \mathrm{D}$ reconstruction technique. An exact matching between the reconstructed surface model and the projections of the femur in the radiographs is observed. a Superimposition of the reconstructed surface model onto the radiographs; b superimposition of the projection of the apparent contours (yellow) of the reconstructed surface model with the X-ray projections of the proximal femur applied to convert a $2 \mathrm{D}-3 \mathrm{D}$ problem to a $3 \mathrm{D}-3 \mathrm{D}$ one. We have designed and conducted experiments using different types of input data to validate the present approach.

The reason why we require a MR-PDM can be summarized as follows. Due to the sparseness of the point data, we need a FL-PDM to establish precise correspondences between each input point and the point on the FL-PDM in the second stage of our 3D-3D reconstruction method, although it is true that this is not an important issue in the first and the third stage of our 3D-3D reconstruction approach. In these two stages we even can use the CL-PDM together with a KD-Tree data structure to accelerate the correspondence establishment procedure. However, if a CL-PDM is used in the second stage, each input point will find a point on the surface model described by the CL-PDM as its corresponding point. However, this point is not necessary a vertex of the CL-PDM. Then to solve the statistical instantiation problem, we either need to use the closest vertex as the correspondence of the input point, or we need to do an interpolation. The former method will bring the correspondence error into the statistical instantiation procedure due to the relative sparseness of the CL-PDM, whereas the latter method will complicate 
Fig. 7 Color-coded error TRE distribution when the reconstructed surface model as shown in Fig. 6 was compared to its ground truth obtained from a CT-scan reconstruction method. The maximum TRE is $5.86 \mathrm{~mm}$, the $95 \%$ percentile TRE is $2.43 \mathrm{~mm}$, and the mean TRE is $0.88 \mathrm{~mm}$. Note that we only computed the TREs from the reconstructed surface model to the ground truth as the former was smaller than the latter
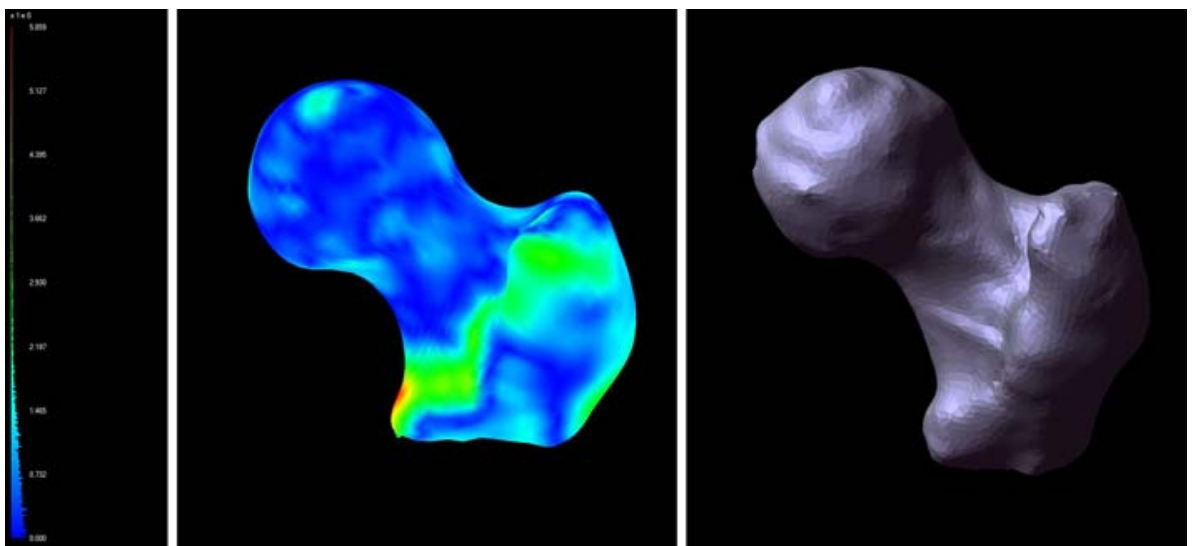

the solution to the statistical instantiation problem. In contrast, this is different when the co-registered images are taken as the input for the 2D-3D reconstruction. Although the number of input images is limited, the extracted edge contours from these images are dense (a sub-pixel resolution). In such a situation, we do not need a FL-PDM to set up a precise correspondence. If a vertex on the PDM is located on the apparent contours of the model and is preserved even after the 2D-3D correspondence establishment procedure, we can always find a 3D point on the forward projection ray of an edge point as its corresponding point. Thus, at each step of the $2 \mathrm{D}-3 \mathrm{D}$ reconstruction, new points on both the PDM and the projection rays of the edge points may be changeable, while in the 3D-3D case the input point set remains the same throughout all iteration.

From the results of the second experiment, one can observe that the accuracy of the present approach can be enhanced with the increase of the number of the input $\mathrm{C}$-arm images. However, considering the cumbersomeness of intraoperative image acquisition, using two calibrated fluoroscopic images as the input is more appropriate for a clinical application.

Our previous work [17] shows that our 3D-3D reconstruction method can handle both non-pathological and pathological cases even when the PDM is constructed from the surface models of normal patients. The results of the third experiment, where the surface models of twenty-three femurs with different shapes have been successfully reconstructed with the present approach, further confirmed that the our 2D-3D reconstruction method can also handle both non-pathological and pathological cases, which is a clear advantage over existing works.

\section{References}

1. Livyatan H, Yaniv Z, Joskowicz L (2003) Gradient-based 2-D/3-D rigid registration of fluoroscopic X-ray to CT. IEEE Trans Med Imaging 22(11):1395-1406
2. Barratt DC, Chan CSK, Edwards PJ, Penney GP, Slomczykowski M, Carter TJ, Hawkes DJ (2008) Instantiation and registration of statistical shape models of the femur and pelvis using 3D ultrasound imaging. Med Image Anal 12:358-374

3. Davies RH, Twining C, Cootes TF, Waterton JC, Taylor CJ (2002) A minimum description length approach to statistical shape modeling. IEEE Trans Med Imaging 21(5):525-537

4. Dalal P, Munsell BC, Wantg S et al (2007) A fast 3D correspondence method for statistical shape modeling. In: Proceedings of CVPR'07, pp 1-8. IEEE Conference on Computer Vision and Pattern Recognition (2007)

5. Zhao Z, Teoh EK (2008) A new scheme for automated 3D PDM construction using deformable models. Image Vis Comput 26: 275288

6. Hufnagel $\mathrm{H}$, Pennec $\mathrm{X}$, Ehrhardt $\mathrm{J}$, Ayache $\mathrm{N}$, Handels $\mathrm{H}$ (2008) Generation of a statistical shape model with probabilistic point correspondences and the expectation maximization-iterative closest point algorithm. Int J Comput Assisted Radiol Surg 2(5): 265-273

7. Styner MA, Rajamani KT, Nolte L-P, Zsemlye G, Székely G, Taylor CJ, Davies RH (2003) Evaluation of 3D correspondence methods for model building. In: Proceedings of IPMI 2003. LNCS, vol 2732. Springer, Berlin, pp 63-75

8. Karlsson J, Ericsson A (2006) A ground truth correspondence measure for benchmarking. In: Proceedings of ICPR 2006, vol 3, pp 568-573

9. Munsell BC, Dalal P, Wang S (2008) Evaluating shape correspondence for statistical shape analysis: a benchmark study. IEEE Trans Pattern Anal Mach Intell 30(11):2023-2039

10. Richard T, Wang S (2005) Nonrigid shape correspondence using landmark sliding, insertion and deletion. In: Proceedings of MICCAI'05, Part II, pp 435-442

11. Chui H, Rangarajan A (2003) A new point matching algorithm for non-rigid registration. Comput Vis Image Underst 89(2/3): 114141

12. Zheng G, Rajamani KT, Nolte L-P (2006) Use of a dense point distribution model in a three-stage anatomical shape reconstruction from sparse information for computer assisted orthopaedic surgery: a preliminary study. In: Proceedings of ACCV 2006, Part II. LNCS, vol 3852, Springer, Berline, pp 52-60

13. Zheng G, Dong X, Nolte L-P (2006) Robust and accurate reconstruction of patient-specific 3D surface models from sparse point sets: a sequential three-stage trimmed optimization approach. In: Proceedings of MIAR 2006. LNCS, vol 4091. Springer, Berlin, pp 68-75

14. Zheng G et al (2007) Accurate and robust reconstruction of a surface model of the proximal femur from sparse point data and a 
dense point distribution model for surgical navigation. IEEE Trans Biomed Eng 54(12):2109-2122

15. Zheng G (2006) A novel 3D/2D correspondence building method for anatomy-based registration. In: Proceedings of WBIR 2006. LNCS, vol 4057, pp 75-83

16. Zheng $\mathrm{G}$ et al (2006) Reconstruction of patient-specific 3D bone surface from 2D calibrated fluoroscopic images and point distribution model. In: Proceedings of MICCAI 2006. LNCS, vol 4190, pp 25-32

17. Zheng G et al (2009) A 2D/3D corresponding building method for reconstruction of a patient-specific 3D bone surface model using point distribution models and calibrated X-ray images. Med Image Anal (accepted for publication)

18. Dryden IL, Mardia KV (1998) Statistical shape analysis. Wiley, New York

19. Kendall D (1989) A survey of the statistical theory of shape. Stat Sci 4(2):87-99

20. Small C (1996) The statistical theory of shape. Springer, Berlin

21. Turk M, Pentland A (1991) Eigenfaces for recognition. J Cogn Neurosci 3(1):71-86

22. Cootes TF et al (1995) Active shape models - their training and application. Comput Vis Image Underst 61(1):38-59

23. Corouge I et al (2003) Interindividual functional mapping: a nonlinear local approach. Neuroimage 19:1337-1348

24. Fleute M, Lavallée S (1998) Building a complete surface model from sparse data using statistical shape models: application to computer assisted knee surgery system. In: Proceedings of MICCAI 1998. LNCS, vol 1496. Springer, Berlin, pp 879-887

25. Fleute M, Lavallée S, Julliard R (1999) Incorporating a statistically based shape model into a system for computer-assisted anterior cruciate ligament surgery. Med Image Anal 3(3):209-222

26. Rajamani KT, Styner M, Joshi SC (2004) Bone model morphing for enhanced surgical visualization. In: Proceedings of the ISBI 2004, pp 1255-1258

27. Rajamani KT et al (2007) Statistical deformable bone models for robust 3D surface extrapolation from sparse data. Med Image Anal 11(2):99-109

28. Fleute M, Lavallée S (1999) Nonrigid 3-D/2-D registration of images using statistical models. In: Proceedings of MICCAI 1999. LNCS, vol 1679. Springer, Belin, pp 138-147

29. Yao J, Taylor RH (2003) Assessing accuracy factors in deformable 2D/3D medical image registration using a statistical pelvis model. In: Proceedings of ICCV 2003, vol 2. IEEE Computer Society, USA, pp 1329-1334

30. Lamecker H, Wenckebach TH, Hege H-C (2006) Atlas-based 3Dshape reconstruction from X-ray images. In: Proceedings of ICPR 2006, vol 1, pp 371-374

31. Benameur $S$ et al (2003) 3D/2D registration and segmentation of scoliotic vertebrae using statistical models. Comput Med Imaging Graph 27:321-337

32. Benameur S et al (2005) A hierarchical statistical modeling approach for the unsupervised 3-D biplanar reconstruction of the scoliotic spine. IEEE Trans Biomed Eng 52(12):2041-2057
33. Chan CSK, Edwards PJ, Hawkes DJ (2003) Integration of ultrasound-based registration with statistical shape models for computer-assisted orthopaedic surgery. In: Proceedings of SPIE Medical Imaging 2003: Image Processing, vol 5032, pp 414-424

34. Chan CSK, Barratt DC, Edwards PJ, Penney GP, Slomczykowski M, Carter TJ, Hawkes DJ (2004) Cadaver validation of the use of ultrasound for 3D model instantiation of bony anatomy in image guided orthopaedic surgery. In: Proceedings of MICCAI 2004, Part II. LNCS, vol 3217. Springer, Berlin, pp 397-404

35. Talib H, Rajamani KT, Kowal J, Nolte L-P, Styner M, Gonzalez Ballester MA (2005) A comparison study assessing the feasibility of ultrasound-initialized deformable bone models. Comput Aided Surg 10(5/6):293-299

36. Talib $\mathrm{H}$ et al (2006) Feasibility of 3D ultrasound-initialized deformable bone-modeling. In: Proceedings of CAOS 2006, pp 519-522

37. Yao J, Taylor RH (2000) Tetrahedral mesh modeling of density data for anatomical atlases and intensity-based registration. In: Proceedings of MICCAI 2000. LNCS, vol 1935. Springer, Berlin, pp 531540

38. Blanz V, Vetter T (1999) A morphable model for the synthesis of 3D faces. In: Proceedings of the 26th annual conference on computer graphics, SIGGRAPH'99, pp 187-194

39. Wang YS et al (2000) Shape-based 3D surface correspondence using geodesics and local geometry. In: CVPR 2000, pp 644-651

40. Davies RH, Twining CH et al (2002) 3D statistical shape models using direct optimization of description length. ECCV'02. LNCS, vol 2352. Springer, Berlin, pp 3-20

41. Loop CT (1987) Smooth subdivision surfaces based on triangles. M.S. Thesis, Department of Mathematics, University of Utah, August

42. Besl P, McKay ND (1992) A method for registration of 3D shapes. IEEE Trans Pattern Anal Mach Intell 14(2):239-256

43. Bookstein F (1989) Principal warps: thin-plate splines and the decomposition of deformations. IEEE Trans Pattern Anal Mach Intell 11(6):567-585

44. Moreland JR (1988) Primary total hip arthroplasty. In: Chapman MW (ed) Operative orthopaedics, vol 1, 1st edn. JB Lippincott, Philadelphia, pp 679-693

45. Schumann S, Zheng G, Nolte L-P (2008) Calibration of X-ray radiographs and its feasible application for $2 \mathrm{D} / 3 \mathrm{D}$ reconstruction of the proximal femur. In: Proceedings of the 30th Annual International IEEE EMBS conference, Vancouver, British Columbia, Canada, 20-24 August, 2008, pp 470-473

46. Zheng G, Schumann S (2008) 3-D reconstruction of a surface model of the proximal femur from digital biplanar radiographs. In: Proceedings of the 30th annual international IEEE EMBS conference, Vancouver, British Columbia, Canada, 20-24 August, 2008, pp 66-69

47. Aspert N, Santa-Cruz D, Ebrahimi T (2002) MESH: measuring error between surface using the Hausdorff distance. In: Proceedings of the IEEE international conference on multimedia and Expo 2002 (ICME) I, pp 705-708 